\title{
TITLE:
}

\section{Structure of the exact wave function. III. Exponential ansatz}

$\operatorname{AUTHOR}(S):$

Nakatsuji, $\mathrm{H}$

CITATION:

Nakatsuji, H. Structure of the exact wave function. III. Exponential ansatz. JOURNAL OF CHEMICAL PHYSICS 2001, 115(6): 2465-2475

ISSUE DATE:

2001-08-08

URL:

http://hdl.handle.net/2433/39733

\section{RIGHT:}

Copyright 2001 American Institute of Physics. This article may be downloaded for personal use only. Any other use requires prior permission of the author and the American Institute of Physics. 


\title{
Structure of the exact wave function. III. Exponential ansatz
}

\author{
Hiroshi Nakatsuji ${ }^{a}$ \\ Department of Synthetic Chemistry and Biological Chemistry, Graduate School of Engineering, \\ Kyoto University, Sakyo-ku, Kyoto 606-8501, Japan
}

(Received 22 January 2001; accepted 22 May 2001)

\begin{abstract}
We continue to study exponential ansatz as a candidate of the structure of the exact wave function. We divide the Hamiltonian into $N_{D}$ (number of divisions) parts and extend the concept of the coupled cluster (CC) theory such that the cluster operator is made of the divided Hamiltonian. This is called extended coupled cluster (ECC) including $N_{D}$ variables (ECCND). It is shown that the $\mathrm{S}$ (simplest)ECC, including only one variable $\left(N_{D}=1\right)$, is exact in the sense that it gives an explicit solution of the Schrödinger equation when its single variable is optimized by the variational or $\mathrm{H}$-nijou method. This fact further implies that the ECCND wave function with $N_{D} \geqslant 2$ should also have a freedom of the exact wave function. Therefore, by applying either the variational equation or the H-nijou equation, ECCND would give the exact wave function. Though these two methods give different expressions, the difference between them should vanish for the exact wave function. This fact solves the noncommuting problem raised in Paper I [H. Nakatsuji, J. Chem. Phys. 113, 2949 (2000)]. Further, ECCND may give more rapidly converging solution than SECC because of its non-linear character, ECCND may give the exact wave function at the sets of variables different from SECC. Thus, ECCND is exact not only for $N_{D}=1$, but also for $N_{D} \geqslant 2$. The operator of the $\mathrm{ECC}, \exp (S)$, is an explicit expression of the wave operator that transforms a reference function into the exact wave function. The coupled cluster including general singles and doubles (CCGSD) proposed in Paper I is an important special case of the ECCND. We have summarized the method of solution for the SECC and ECCND truncated at order $n$. The performance of SECC and ECC2 is examined for a simple example of harmonic oscillator and the convergence to the exact wave function is confirmed for both cases. Quite a rapid convergence of ECC2 encourages an application of the ECCND to more general realistic cases. (c) 2001 American Institute of Physics.
\end{abstract}

[DOI: 10.1063/1.1385371]

\section{INTRODUCTION}

A purpose of this series of studies ${ }^{1,2}$ is to clarify the structure of the exact wave function. Since the Hamiltonian in the Schrödinger equation is simple, involving the operators only up to two-particle interaction terms, the exact wave function, its eigenfunction, should also have some simple structure, reflecting this simplicity of the Hamiltonian operator. We have already shown in the previous two papers ${ }^{1,2}$ that certainly the exact wave function can be described with a smaller number of variables than ever thought. After clarifying the structure of the exact wave function, we study the efficient method to calculate it. The Schrödinger equation governs essentially most of physics and all of chemistry, ${ }^{3}$ so that if we can solve this equation with a realistic cost, we can make very precise predictions and its scientific and practical merits are huge.

The Schrödinger equation is given by

$$
(H-E) \psi=0 \text {, }
$$

where the Hamiltonian involves only one- and two-particle operators as

$$
H=\sum_{i}^{N} v(i)+\sum_{i>j}^{N} w(i, j),
$$

${ }^{a)}$ Electronic mail: hiroshi@sbchem.kyoto-u.ac.jp in a coordinate representation, or

$$
H=\sum_{p r} v_{p}^{r} a_{r}^{+} a_{p}+\sum_{p q r s} w_{p q}^{r s} a_{r}^{+} a_{s}^{+} a_{q} a_{p},
$$

in a second-quantized representation. The most straightforward method of solving the Schrödinger equation within a given basis set is the full CI method, but the number of the variables, $M_{\text {full-CI }}$ involved in this method

$$
M_{\text {full-Cl }}=\frac{1}{m+1}\left(\begin{array}{c}
m+1 \\
\frac{1}{2} N
\end{array}\right)\left(\begin{array}{c}
m+1 \\
\frac{1}{2} N+1
\end{array}\right),
$$

easily runs into astronomical figures even for small molecules. In Eq. (1.3) $m$ is the number of active orbitals, $N$ the number of electrons, and () denotes binomial coefficient.

In the first paper of this series, ${ }^{1}$ hereafter called Paper I, we have shown that it is possible to solve the Schrödinger equation with $M_{\mathrm{GSD}}$ variables

$$
M_{\mathrm{GSD}}=m^{2}+\left[\frac{m}{2}(m-1)\right]^{2} .
$$

This is the number of the general singles and doubles (GSD) substitution operators and is much smaller than $M_{\text {full-CI }}$. We have proposed the iterative configuration interaction (ICI) method involving GSD number of variables (ICIGSD) as a 
method to calculate the exact wave function. In the second paper of this series, ${ }^{2}$ called Paper II, we have shown that an even smaller number of variables is possible to solve the Schrödinger equation. The number of variables depends on how we divide the Hamiltonian operator into pieces. This number of division, $N_{D}$ is, for example, from unity to $M_{\mathrm{GSD}}$ : unity is for no division and $M_{\mathrm{GSD}}$ for dividing $H$ into all the terms in Eq. (1.2b). This generalized ICI method was called the ICIND method. Further, we have described how to calculate the excited states from the ICIND method.

In Paper I, we have obtained the theorem that suggests the possibility that the exponential anzatz may represent the structure of the exact wave function when it is combined with the variational principle. The coupled cluster singles (CCS) is certainly exact when the Hamiltonian involves only a single particle operator. However, the conventional CCSD was shown to be not exact because it is not variational and because the operators involved are only excitation-operator type ( ${ }^{\mathrm{A}} t$ type) and do not include other substitution operators ( ${ }^{\mathrm{X}} t$ type). ${ }^{1}$ We then proposed the CCGSD method that was the CC including general singles and doubles (GSD) substitution operators, but the problem was that it included the noncommuting operators. Nooijen ${ }^{8}$ recently considered a combination of CCGSD with the density equation. ${ }^{5}$

In this paper, we will extend the exponential ansatz in the light of the general theorem given in Paper II and present extended coupled cluster (ECC) theory. We will show that the ECC in its simplest form (SECC: simplest extended coupled cluster) certainly represents the structure of the exact wave function as expected originally in Paper I. This fact further sheds light on the structure of the ECC theory in general. We analyze the structure of the ECC wave function. A method of solving the ECC is described and some simple application is given.

The wave operator $W$ is an operator that transforms an appropriate approximate wave function $\psi_{0}$ into the exact wave function $\psi$ that is a solution of the Schrödinger equation

$\psi=W \psi_{0}$.

When $\psi_{0}$ is Hartree-Fock, $W$ includes all the correlation effects. We will give in this paper a simple explicit expression of the wave operator based on the exponential ansatz.

\section{BACKGROUND}

We first summarize several equations that are equivalent to the Schrödinger equation in the necessary and sufficient sense. They provide valuable information for the study of the structure of the exact wave function, because they have the same determinative power as the Schrödinger equation when they are solved appropriately.

The most well-known and useful one is the variational principle

$$
\langle\psi|H-E| \delta \psi\rangle=0,
$$

where the energy of the system, $E$, is defined by

$$
\langle\psi|H-E| \psi\rangle=0 .
$$

Second, the equation

$$
\left\langle\psi\left|(H-E)^{2}\right| \psi\right\rangle=0
$$

is equivalent to the Schrödinger equation, and each of the following two equations,

$$
\langle\psi|(H-E) H| \psi\rangle=0,
$$

and

$$
\left\langle\psi\left|H^{2}-E^{2}\right| \psi\right\rangle=0,
$$

together with Eq. (2.2) is also equivalent to the Schrödinger equation. ${ }^{5}$

Based on these equations, we have given the following theorem in Paper II. Namely, when we divide the Hamiltonian into the $N_{D}$ parts as

$$
H=\sum_{I=1}^{N_{D}} H_{I},
$$

then, the wave function $\psi$ that satisfies

$$
\left\langle\psi\left|(H-E) H_{I}\right| \psi\right\rangle=0 \quad\left(I=1, \ldots, N_{D}\right),
$$

and Eq. (2.2) is exact in a necessary and sufficient sense. Similarly, the wave function $\psi$ that satisfies

$$
\left\langle\psi\left|(H-E)\left(H_{I}-E_{I}\right)\right| \psi\right\rangle=0 \quad\left(I=1, . ., N_{D}\right),
$$

is also exact in the necessary and sufficient sense. In Eq. (2.8), the partial energy $E_{I}$ corresponding to $H_{I}$ is defined by

$$
\left\langle\psi\left|\left(H_{I}-E_{I}\right)\right| \psi\right\rangle=0,
$$

and satisfies

$$
E=\sum_{I} E_{I}
$$

It is convenient to call this group of equations H-square or $\mathrm{H}$-nijou equations. ("Nijou" is Japanese meaning square.)

Based on the above theorem, we have introduced in $\mathrm{Pa}$ per II the iterative CI method including $N_{D}$ variables (ICIND) as a method that gives the exact wave function. Actually, we can obtain the exact wave function, corresponding to any division of the Hamiltonian. For example, we can obtain the exact wave function using only one variable. We can define the exact wave function including only the singles number (number of singles substitution operators) of variables: this is an interesting special case that may give a basis of the density functional theory (DFT). ${ }^{6}$ When we divide the Hamiltonian into the GSD parts, as in the expression of Eq. (1.2b), we have the ICIGSD method proposed in Paper I. Since each iteration process of the ICI method is variational, the solution converges from above to the exact wave function. We have explained further in Paper II how to calculate the excited states within the framework of the ICI method.

\section{EXTENDED COUPLED CLUSTER}

In Paper I, we have examined the exponential ansatz as a promising candidate of the structure of the exact wave function. This expectation was based on Theorem II-2 of Paper I that suggested that the exponential ansatz might represent the structure of the exact wave function. We study this problem here in the light of the theorem given in Paper II. 
We describe here an extension of the coupled cluster (CC) theory. Corresponding to the division of the Hamiltonian

$$
H=\sum_{I=1}^{N_{D}} H_{I},
$$

we define the variable operator

$$
S=\sum_{I=1}^{N_{D}} C_{I} H_{I},
$$

which includes $N_{D}$ variables $\left\{C_{I}\right\}$. Using this variable operator, we define the coupled cluster expansion by

$$
\begin{aligned}
\psi & =\exp (S) \psi_{0} \\
& =\left[1+S+\frac{1}{2} S^{2}+\frac{1}{3 !} S^{3}+\cdots\right] \psi_{0},
\end{aligned}
$$

where $\psi_{0}$ is some reference function that is usually HartreeFock, $|0\rangle$. This is a small extension of the coupled cluster method, so we call it extended coupled cluster (ECC) method. Following the conventional notation, we call the ECC given by Eq. (3.3) ECCND (ECC with $N_{D}$ variables) method. Another variation of ECCND is introduced by defining the variable operator $S$ by

$$
S=\sum_{I=1}^{N_{D}} c_{I}\left(H_{I}-E_{I}\right)
$$

where $E_{I}$ is given by Eq. (2.9) and satisfies Eq. (2.10). The wave function is commonly written by Eq. (3.3).

Note that the coupled cluster general singles and doubles (CCGSD) defined by ${ }^{1}$

$$
\psi=\exp (T) \psi_{0},
$$

with

$$
\begin{aligned}
T & =\sum_{p r} C_{p}^{r} a_{r}^{+} a_{p}+\sum_{p q r s} C_{p q}^{r s} a_{r}^{+} a_{s}^{+} a_{q} a_{p} \\
& =\sum_{p r} c_{p}^{r} v_{p}^{r} a_{r}^{+} a_{p}+\sum_{p q r s} c_{p q}^{r s} w_{p q}^{r s} a_{r}^{+} a_{s}^{+} a_{q} a_{p},
\end{aligned}
$$

is a special case of the ECCND: we use the Hamiltonian (1.2b) in defining the $S$ operator (here, $T$ operator) having GSD number of variables.

The CCGSD in the form of Eq. (3.4) is written as follows. We define the partial energies $E_{p}^{r}$ and $E_{p q}^{r s}$ by

$$
\begin{aligned}
& \left\langle\psi\left|v_{p}^{r} a_{r}^{+} a_{p}-E_{p}^{r}\right| \psi\right\rangle=0, \\
& \left\langle\psi\left|w_{p q}^{r s} a_{r}^{+} a_{s}^{+} a_{q} a_{p}-E_{p q}^{r s}\right| \psi\right\rangle=0,
\end{aligned}
$$

and the density matrices $n_{p}^{r}$ and $n_{p q}^{r s}$ by

$$
\left\langle\psi\left|a_{r}^{+} a_{p}-n_{p}^{r}\right| \psi\right\rangle=0, \quad\left\langle\psi\left|a_{r}^{+} a_{s}^{+} a_{q} a_{p}-n_{p q}^{r s}\right| \psi\right\rangle=0,
$$

where

$$
E_{p}^{r}=v_{p}^{r} n_{p}^{r}, \quad E_{p q}^{r s}=w_{p q}^{r s} n_{p q}^{r s} .
$$

The partial energies sum up to $E$ as

$$
E=\sum_{p, r} E_{p}^{r}+\sum_{p q, r s} E_{p q}^{r s},
$$

and the density matrices to

$$
N=\operatorname{Tr}\left\{n_{p}^{r}\right\}, \quad N(N-1)=\operatorname{Tr}\left\{n_{p q}^{r s}\right\},
$$

where $N$ is the number of electrons. Using these quantities, we can define the CCGSD in the form of

$\psi=\exp \left(T^{\prime}\right) \psi_{0}$,

$T^{\prime}=\sum_{p, r} D_{p}^{r}\left(1-n_{p}^{r}\right) a_{r}^{+} a_{p}+\sum_{p q r s} D_{p q}^{r s}\left(1-n_{p q}^{r s}\right) a_{r}^{+} a_{s}^{+} a_{q} a_{p}$

$$
=\sum_{p r} d_{p}^{r}\left(v_{p}^{r}-E_{p}^{r}\right) a_{r}^{+} a_{p}+\sum_{p q r s} d_{p q}^{r s}\left(w_{p q}^{r s}-E_{r q}^{r s}\right) a_{r}^{+} a_{s}^{+} a_{q} a_{p},
$$

where $D_{p}^{r}, D_{p q}^{r s}$ and $d_{p}^{r}, d_{p q}^{r s}$ represent the variables.

We study below the ECCND, classifying it into the two cases, namely the simplest ECC (SECC) with $N_{D}=1$ and the general ECCND with $N_{D} \geqslant 2$.

\section{A. SECC}

We first consider the simplest extended coupled cluster (SECC), where $N_{D}=1$ in Eq. (3.1); namely, we do not divide the Hamiltonian operator. Then, SECC may also be referred to as ECC1 as an extreme of the ECCND. The $S$ operator of the type of Eq. (3.2) is written as $S=C H$, and the SECC wave function is written as

$$
\begin{aligned}
\psi & =\exp (C H) \psi_{0} \\
& =\left(1+C H+\frac{1}{2} C^{2} H^{2}+\frac{1}{3 !} C^{3} H^{3}+\cdots\right) \psi_{0} .
\end{aligned}
$$

We calculate the energy $E$ by $\langle\psi|H-E| \psi\rangle=0$ and the variable $C$ by the variational principle. Differentiating $\psi$ with respect to $C$, we obtain

$$
\frac{\partial \psi}{\partial C}=H \psi
$$

Inserting this relation into the variational equation, we have

$$
\langle\psi|(H-E) H| \psi\rangle=0,
$$

which is Eq. (2.4). Therefore, the SECC given by Eq. (3.14) is exact when it is solved variationally.

Using the $S$ operator of the type of Eq. (3.4) as $S$ $=c(H-E)$, the SECC can be written in a different form

$$
\begin{aligned}
\psi & =\exp [c(H-E)] \psi_{0} \\
& =\left[1+c(H-E)+\frac{1}{2} c^{2}(H-E)^{2}+\frac{1}{3 !} c^{3}(H-E)^{3}+\cdots\right] \psi_{0},
\end{aligned}
$$

which satisfies 


$$
\frac{\partial \psi}{\partial c}=(H-E) \psi
$$

Therefore, from the variational principle, we have

$$
\left\langle\psi\left|(H-E)^{2}\right| \psi\right\rangle=0,
$$

which is Eq. (2.3). Therefore, the variational SECC in the form of Eq. (3.17) is also exact.

Thus, we have the theorem.

Theorem III: Variational SECC is an explicit expression of the solution of the Schrödinger equation.

Theorem III is rather surprising. It means that the variational SECC is exact, though it has only one variable. It also means that the operator of the $\mathrm{SECC}, \exp (\mathrm{CH})$ or $\exp [c(H$ $-E)$ ], is an explicit expression of the wave operator defined by Eq. (1.5). It transforms an approximate wave function $\psi_{0}$ into the exact one $\psi$. Since the operator, $\exp (\mathrm{CH})$ or $\exp [c(H-E)]$, is totally symmetric, the wave function of SECC reflects the symmetry of the reference function $\psi_{0}$. This implies that the SECC is applicable to the ground state of each spin-space symmetry.

The convergence of SECC, when it is written in an expansion form as in Eq. (3.14b) or (3.17b), is clear, since a merit of the exponential ansatz is that it always converges, though this does not necessarily mean that the convergence is fast. The convergence rate is dependent on the quality of the reference function $\psi_{0}$. It is very much desirable to calculate the SECC wave function, $\exp (\mathrm{CH}) \psi_{0}$ or $\exp [c(H$ $-E)] \psi_{0}$, directly as it is in Eq. (3.14a) or (3.17a) rather than expanding it as in Eq. (3.14b) or (3.17b).

There is no iteration in the structure of the SECC, different from the ICI method. In comparison with the simplest ICI (SICI) given by Eq. (3.18) or (3.19) of Paper II, the SECC has uniquely only one variable, while in the SICI method, the single variable is reoptimized in each iteration step.

The SECC given by Eq. (3.17) is somewhat more transparent than that of Eq. (3.14). When $\psi_{0}$ is an eigenfunction of $H$, then $\exp [c(H-E)]=1$, and therefore, $\psi=\psi_{0}$ as it should be. When $\psi_{0}$ is close to $\psi,(H-E)$ should be small and therefore the convergence should be fast. Actually, however, the operators, $\exp (\mathrm{CH})$ and $\exp [c(H-E)]$, are essentially the same: the difference lies only in the norm of the wave function.

Solution of the Schrödinger equation in the form of SECC has long been considered in the field of quantum molecular dynamics dealing with the time-dependent Schrödinger equation. ${ }^{7,8}$ Kozloff and Tal-Ezer, ${ }^{8}$ for example, considered the solution of the time-dependent Schrödinger equation on the imaginary time axis. Then, the solution of the time-dependent Schrödinger equation becomes

$$
\psi(\tau)=\exp (-H \tau) \psi(0),
$$

where $\tau=i t$ with $t$ being (real) time. In comparison with Eq. (3.14a), the correspondence is $C=-\tau, \psi=\psi(\tau)$, and $\psi_{0}$ $=\psi(0)$. This $\psi(\tau)$ approaches the exact wave function of the ground state of $H$ after sufficiently long time interval starting from an initial function $\psi(0)$. Expanding the initial wave function $\psi(0)$ in terms of the eigenfunctions $\left\{\Psi_{i}\right\}$ of $H$ (the ordering of the states is $E_{0}<E_{1}<E_{2}<\ldots$ ), we obtain ${ }^{8}$

$$
\psi(\tau)=\exp (-H \tau) \sum_{i} d_{i} \Psi_{i}(0)=\sum_{i} d_{i} \exp \left(-E_{i} \tau\right) \Psi_{i}(0)
$$

After a time $\tau$, the eigenfunction $\Psi_{i}$ is reduced relative to $\Psi_{0}$ by the ratio, $\exp \left[-\left(E_{i}-E_{0}\right) \tau\right]$, so that after the time $\tau$ larger than $m \ln 10 /\left(E_{1}-E_{0}\right), \psi(\tau)$ approaches $\Psi_{0}$ in $m$ digits of accuracy. At $\tau$ infinity, $\psi(\tau)$ becomes equal to $\Psi_{0}$. As $\left\{\Psi_{i}\right\}$ are all linearly independent, this is a unique way of getting the exact solution from Eq. (3.21).

This argument means that the variable $C$ or $c$ in the SECC is a large negative (infinite) value to represent the exact wave function. Of course, the wave function itself is finite when it is divided by the normalization factor. The actual optimal value is dependent on the quality of the reference wave function $\psi_{0}$. We will see in the applications of SECC that the actual optimal value of the variable is not so large negative, as far as we solve it approximately.

The most important conclusion of this section is that the simplest ECC, SECC, has a structure of the exact wave function and can certainly become exact with its structure of the exponential ansatz.

\section{B. ECCND}

We next consider the general case of the ECCND where $N_{D} \neq 1$. Now, we know that even the simplest form of the exponential ansatz, SECC, has the structure of the exact wave function, so that we can assume that a more general exponential ansatz, ECCND, does have freedom to represent the structure of the exact wave function. Therefore, when we calculate the variables $\left\{C_{I}\right\}$ in Eq. (3.2) either by the variational method or by the H-nijou method, both give the exact wave function, because both methods are equivalent to the Schrödinger equation in the necessary and sufficient sense.

We first apply the variational principle to the ECCND wave function given by Eq. (3.3). Since the operators $\left\{H_{I}\right\}$ in Eq. (3.2) do not generally commute with each other

$$
\left[H_{I}, H_{J}\right] \neq 0 \quad(I \neq J),
$$

the variation of $\psi$ with respect to $C_{I}$ is written as

$$
\begin{aligned}
\frac{\partial \psi}{\partial C_{I}}= & {\left[H_{I}+\frac{1}{2}\left(H_{I} S+S H_{I}\right)+\frac{1}{3 !}\left(H_{I} S^{2}+S H_{I} S+S^{2} H_{I}\right)\right.} \\
& +\cdots] \psi_{0},
\end{aligned}
$$

where the $S$ operator is given by Eq. (3.2), and we have used the relation

$$
\frac{\partial S}{\partial C_{I}}=H_{I}
$$


Equation (3.23) means that $\partial \psi / \partial C_{I} \neq H_{I} \psi$ in general. Combining Eq. (3.23) with the variational principle given by Eq. (2.1), we obtain the variational working equation for the ECCND method as

$$
\begin{aligned}
G^{I}\left(\left\{C_{I}\right\}\right)=\langle\psi|(H-E)\left[H_{I}+\frac{1}{2}\left(H_{I} S+S H_{I}\right)\right. \\
\left.+\frac{1}{3 !}\left(H_{I} S^{2}+S H_{I} S+S^{2} H_{I}\right)+\cdots\right]\left|\psi_{0}\right\rangle=0,
\end{aligned}
$$

for all $I$. Note that this equation is highly nonlinear for the variable $\left\{C_{I}\right\}$. Multiplying $C_{I}$ in Eq. (3.25) and summing up for $I$, we obtain

$$
\langle\psi|(H-E) S| \psi\rangle=0 .
$$

A simple sum of Eq. (3.25) for all $I$ gives

$$
\begin{aligned}
& \langle\psi|(H-E)\left[H+\frac{1}{2}(H S+S H)\right. \\
& \left.\quad+\frac{1}{3 !}\left(H S^{2}+S H S+S^{2} H\right)+\cdots\right]\left|\psi_{0}\right\rangle=0 .
\end{aligned}
$$

Next, we apply the H-nijou method to the ECCND wave function. The H-nijou method is defined by Eqs. (2.7) or (2.8). Inserting the ECCND wave function into Eq. (2.7), we have the working equation of the H-nijou method as

$$
\begin{aligned}
F^{I}\left(\left\{C_{I}\right\}\right) & =\left\langle\psi\left|(H-E) H_{I}\right| \psi\right\rangle \\
& =\left\langle\psi\left|(H-E)\left[H_{I}+H_{I} S+\frac{1}{2} H_{I} S^{2}+\cdots\right]\right| \psi_{0}\right\rangle=0,
\end{aligned}
$$

for all $I$, where $E$ is defined by Eq. (2.2). Equation (3.28) is again nonlinear for the variable $\left\{C_{I}\right\}$.

We see that Eq. (3.25) of the variational method and Eq. (3.28) of the H-nijou method are different. But, since the exact wave function should satisfy both variational and $\mathrm{H}$-nijou equations, it must also satisfy the equation

$$
\begin{aligned}
\Delta^{I}\left(\left\{C_{I}\right\}\right)=\langle & \psi \mid(H-E)\left[\frac{1}{2}\left(H_{I} S-S H_{I}\right)+\frac{1}{3 !}\left(2 H_{I} S^{2}\right.\right. \\
& \left.\left.-S H_{I} S-S^{2} H_{I}\right)+\cdots\right]\left|\psi_{0}\right\rangle=0
\end{aligned}
$$

which is derived by subtracting Eq. (3.25) from Eq. (3.28). This equation can also be written as

$$
\begin{aligned}
\Delta^{I}\left(\left\{C_{I}\right\}\right)=\langle\psi|(H-E)\left\{\frac{1}{2}\left[H_{I}, S\right]+\frac{1}{3 !}\left(2\left[H_{I}, S\right] S\right.\right. \\
\\
\left.\left.+S\left[H_{I}, S\right]\right)+\cdots\right\}\left|\psi_{0}\right\rangle=0
\end{aligned}
$$

and a simple sum of this equation for all $I$ gives

$$
\begin{gathered}
\Delta\left(\left\{C_{I}\right\}\right)=\langle\psi|(H-E)\left\{\frac{1}{2}[H, S]+\frac{1}{3 !}(2[H, S] S\right. \\
+S[H, S])+\cdots\}\left|\psi_{0}\right\rangle=0 .
\end{gathered}
$$

Conversely, when $\Delta^{I}\left(\left\{C_{I}\right\}\right)=0$ together with $G^{I}\left(\left\{C_{I}\right\}\right)=0$ or $F^{I}\left(\left\{C_{I}\right\}\right)=0$ for all $I$, the variational equation (3.25) becomes equal to the H-nijou equation (3.28) for all $I$, and therefore, this $\psi$ must be exact from the theorem given in Paper II.

$\Delta^{I}\left(\left\{C_{I}\right\}\right)=0$, Eq. (3.30), is a highly nonlinear equation for the variables $\left\{C_{I}\right\}$, likewise the variational equation (3.25) and the H-nijou equation (3.28), and may be satisfied in two ways. Case $\mathrm{A}$ is when all $H_{I}$ and $S$ commute, $\left[H_{I}, S\right]=0$. Equation (3.31) is satisfied if $H$ and $S$ commute, $[H, S]=0$. For Eq. (2.4) being held, only $[H, S]=0$ suffices. However, we feel that the commutation relation $[H, S]=0$ is a rather too strong condition in the case of $N_{D} \geqslant 2$. A milder condition is case B, in which Eq. (3.30) is satisfied as it is as an integral relation, even though the above commutation relation does not hold.

It is true that the relation $[H, S]=0$ holds if all $C_{I}=C$ : then, this ECCND reduces to the SECC and therefore its variational solution should be exact. If $H_{I}$ is truly a part of $H$, not like $d_{I} H$ with $d_{I}$ being a proportionality constant, then $H_{I}$ never commutes with $H$, and therefore $[H, S]=0$ holds only when all $C_{I}=C$. Thus, the solution in case A is identical with that of SECC. On the other hand, case B may be satisfied even if $H$ and $S$ do not commute, and therefore even if all $C_{I}$ are not equal. Since the $\psi$ at case B satisfies $\Delta^{I}$ $=0$ together with $G^{I}=0$ or $F^{I}=0$, this $\psi$ must be exact. When case A and case B occur simultaneously at different sets of $\left\{C_{I}\right\}$, giving the same energy, the solution of ECCND is not unique. Since $\Delta^{I}=0$ is a nonlinear equation for $\left\{C_{I}\right\}$, this is possible. Note, however, that since the exact wave function is unique, the solutions corresponding to case $\mathrm{A}$ and $\mathrm{B}$ must be identical except for a normalization factor.

Since case A is identical to SECC, it is realized only when all terms in the expansion of Eq. (3.3b) are included: it must be strictly $\exp (S)$ as the argument of Kosloff and Tal-Ezar $^{8}$ given below Eq. (3.21) implies. A merit of case B over case $\mathrm{A}$ is that case $\mathrm{B}$ can be realized within shorter terms of expansion of the exponential operator, the contribution of the latter's rest of terms being smaller than a given threshold of accuracy: this is realized since ECCND has a larger number of variables than SECC, which has only one variable. For example, let us image CCGSD. There, the variables associated with the ${ }^{X_{t}}$ type operators can become smaller than those of the ${ }^{A} t$-type ones as a result of the variational principle. (Within GSD operators, ${ }^{A} t$ type operators are ordinary excitation operators that appear in CCSD, while the ${ }^{X} t$ type operators are the rest of the GSD operators including de-excitation operators, etc. ${ }^{1}$ ) This is natural since we know that CCSD is already a good approximation of the exact wave function. However, in the SECC only one variable exists, so that such a fine tuning is impossible and a large number of terms, i.e., true exponential operator, is necessary to represent the exact wave function.

In an approximate method of ECCND in which we terminate the expansion of the exponential operator at some order, case B would be realized within some accuracy and give a lower solution than case A. When we have a larger number of variables in ECCND, the convergence rate would 
be faster. This would be especially so when we perform CCGSD, a special case of ECC given in Paper I. ${ }^{9}$ We will see in Sec. $V$ that in a simple example of application, the ECCND gives much faster convergence than SECC because of the realization of case B. In SECC, only case A exists.

Now, it would be interesting if we can introduce the exponential ansatz including only commutable operators. Such formulation is certainly possible if we introduce a normal-ordered exponential ansatz ${ }^{10,11}$

$$
\psi=\{\exp (T)\} \psi_{0},
$$

where the brace \{\} means that we include only the normalordered products in the expansion of the exponential operator. $T$ is the GSD operator given by Eq. (3.6). This expansion does not include the noncommuting operators by its definition. This formulation has been considered first by Lindgren to circumvent noncommuting problems in the formulation of open-shell coupled cluster theory. ${ }^{10}$ Mukherjee also gave some interesting formulations. ${ }^{11}$ When we apply the variational principle to the $\psi$ given by Eq. (3.32), we obtain

$$
\left\langle\psi\left|(H-E)\left\{a_{r}^{+} a_{p} \exp (T)\right\}\right| \psi_{0}\right\rangle=0,
$$

and

$$
\left\langle\psi\left|(H-E)\left\{a_{r}^{+} a_{s}^{+} a_{q} a_{p} \exp (T)\right\}\right| \psi_{0}\right\rangle=0,
$$

but these equations are different from Eqs. (2.1) and (2.2) of Paper I. Therefore, the normal-ordered exponential anzatz given by Eq. (3.32) is not guaranteed to represent the structure of the exact wave function.

Finally, it may be useful to give the formulas for the nonvariational solution of the ECCND. They are given by

$$
\left\langle\psi_{0}|H-E| \psi\right\rangle=0,
$$

and

$$
\left\langle\psi_{0}\left|H_{I}^{*}(H-E)\right| \psi\right\rangle=0 \quad\left(I=1, \ldots, N_{D}\right) .
$$

When $\psi_{0}$ is Hartree-Fock, $H_{I} \psi_{0}$ includes only up to two particle excitations, so that the calculations of Eq. (3.36) is easier than that of Eq. (3.25). Note that when $H_{I}$ includes only ${ }^{X} t$-type operators, most of their coefficients $C_{I}$ may vanish identically as a result of Tables III and IV of Paper I. In contrast to the variational method, this method of solution does not guarantee the solution to be exact. This nonvariational formulation is also valid to the SECC with $N_{D}=1$.

The formulations given here is based on the $S$ operator given by Eq. (3.2). Those based on the $S$ operator given by Eq. (3.4) are easy, therefore, we do not give them for the sake of brevity. The arguments about the ECCND are valid also for this case.

\section{SOLVING ECC}

\section{A. Solving SECC}

We now formulate the basic equations for solving the ECC. Again, we describe the case of SECC first and then the case of ECCND with $N_{D} \geqslant 2$. The main difference lies in the commutation relation.
The working equation for the SECC given by Eq. (3.14a) or (3.17a) is written as

$$
g(C)=\langle\psi|(H-E) H| \psi\rangle=0,
$$

or

$$
f(C)=\left\langle\psi\left|(H-E)^{2}\right| \psi\right\rangle=0,
$$

with $E$ defined by Eq. (2.2). Equations (4.1) and (4.2) are equivalent when the energy $E$ is defined by Eq. (2.2). $C$ in Eqs. (4.1) and (4.2) stands for either $C$ or $c$ in Eq. (3.14a) or (3.17a), respectively. For SECC, the variational and H-nijou equations are the same. If we define

$$
\lambda=(H-E) \exp (C H) \psi_{0},
$$

or

$$
\lambda=(H-E) \exp [c(H-E)] \psi_{0},
$$

then Eq. (4.2) is written as

$$
f(C)=\langle\lambda \mid \lambda\rangle=0 .
$$

Note that always $f(C) \geqslant 0$ and the solution is at the extreme, $f(C)=0$. It is very useful if we can calculate the integral $f(C)$ directly from Eq. (3.14a) or (3.17a) without expanding the exponential operator as in Eq. (3.14b) or (3.17b).

Another method of calculating the SECC may be performed asymptotically by increasing term by term along the expansion of the exponential operator as in Eq. (3.14b) or (3.17b). We truncate the expansion of the exponential operator at the term $(1 / n !) C^{n} H^{n}$ or $(1 / n !) c^{n}\left(H-E_{n}\right)^{n}$ : such truncated wave function is denoted as $\psi_{n}$

$\psi_{n}=\left(1+C H+\frac{1}{2} C^{2} H^{2}+\frac{1}{3 !} C^{3} H^{3}+\cdots+\frac{1}{n !} C^{n} H^{n}\right) \psi_{0}$,

or

$$
\begin{aligned}
\psi_{n}= & {\left[1+c\left(H-E_{n}\right)+\frac{1}{2} c^{2}\left(H-E_{n}\right)^{2}+\frac{1}{3 !} c^{3}\left(H-E_{n}\right)^{3}+\cdots\right.} \\
& \left.+\frac{1}{n !} c^{n}\left(H-E_{n}\right)^{n}\right] \psi_{0}
\end{aligned}
$$

where $E_{n}$ is defined by

$$
\left\langle\psi_{n}\left|H-E_{n}\right| \psi_{n}\right\rangle=0 .
$$

The optimal value of the variable $C$ or $c$ is calculated for the truncated $\psi_{n}$ and the energy and other properties are calculated. Then, we increase $n$, repeat the calculation, and check the convergence. Though the expansion should always converge, the convergence rate would be dependent on the quality of the reference function.

The optimum value of $C$ or $c$ is calculated by the following methods. We define $f_{n}(C)$ from the truncated wave functions given by Eq. (4.6) or (4.7) as

$$
f_{n}(C)=\left\langle\psi_{n}\left|\left(H-E_{n}\right)^{2}\right| \psi_{n}\right\rangle,
$$

and impose the condition 


$$
\operatorname{Min}\left[f_{n}(C)\right], \quad \text { or } \quad \frac{d}{d C} f_{n}(C)=0 .
$$

For the truncated $\psi_{n}, f_{n}(C)$ is positive nonzero. By adding term by term, the function $f_{n}(C)$ approaches zero from above and when it becomes zero, i.e., at the convergence, the corresponding $\psi_{n}$ is exact. This method is called the $f(C)$-minimization method and is different from the variational method described below.

We also calculate the truncated wave functions $\psi_{n}$ by applying the variational principle. Differentiating $\psi_{n}$ by the variable $C$ or $c$, we obtain

$$
\frac{d \psi_{n}}{d C}=H \psi_{n-1},
$$

or

$$
\frac{d \psi_{n}}{d c}=\left(H-E_{n}\right) \psi_{n-1},
$$

for the truncated SECC given by Eq. (4.6) or (4.7), respectively. Inserting these expressions into the variational principle given by Eq. (2.1), we obtain

$$
\left\langle\psi_{n}\left|\left(H-E_{n}\right) H\right| \psi_{n-1}\right\rangle=0,
$$

or

$$
\left\langle\psi_{n}\left|\left(H-E_{n}\right)^{2}\right| \psi_{n-1}\right\rangle=0,
$$

for the truncated SECC. $E_{n}$ is defined by Eq. (4.8). The optimal value of $C$ or $c$ is calculated from Eq. (4.13) or (4.14).

Equations (4.9) and (4.14) show the difference between the $f(C)$-minimization method and the variational method, but we note that they become close as $n$ increases and finally become identical. Before then, the energy calculated by the variational method is lower than that obtained by the $f(C)$-minimization method.

The SECC has some similarity to the solution of the time-dependent Schrödinger equation on the imaginary time axis. Therefore, the method developed for solving the timedependent Schrödinger equation ${ }^{7,8}$ might also be useful for the SECC.

\section{B. Solving ECCND}

For ECCND with $N_{D} \geqslant 2$, the variational equation and the H-nijou equation are different. The method of solution for the exact case is presented in the previous section. We consider here the method of solution for the ECCND truncated at order $n$

$$
\psi_{n}=\left(1+S+\frac{1}{2} S^{2}+\frac{1}{3 !} S^{3}+\cdots+\frac{1}{n !} S^{n}\right) \psi_{0} .
$$

Since this $\psi_{n}$ is an approximation to the ECCND, the variational and the H-nijou methods give different solutions in general, but the difference should be small for good $\psi_{n}$.

The variational working equation is written as

$$
\begin{aligned}
G_{n}^{I}\left(\left\{C_{I}\right\}\right)= & \left\langle\psi_{n}\right|\left(H-E_{n}\right)\left[H_{I}+\frac{1}{2}\left(H_{I} S+S H_{I}\right)\right. \\
& +\frac{1}{3 !}\left(H_{I} S^{2}+S H_{I} S+S^{2} H_{I}\right)+\cdots \\
& \left.+\frac{1}{n !} \sum_{m=1}^{n} S^{m-1} H_{I} S^{n-m}\right]\left|\psi_{0}\right\rangle=0,
\end{aligned}
$$

with $E_{n}$ defined by

$$
\left\langle\psi_{n}\left|H-E_{n}\right| \psi_{n}\right\rangle=0 .
$$

The energy $E_{n}$ becomes minimum for the solution of the ground state.

The working equation for the H-nijou method is given by

$$
\begin{aligned}
F_{n}^{I}\left(\left\{C_{I}\right\}\right)= & \left\langle\psi_{n}\left|\left(H-E_{n}\right) H_{I}\right| \psi_{n}\right\rangle \\
= & \left\langle\psi_{n}\right|\left(H-E_{n}\right)\left(H_{I}+H_{I} S+\frac{1}{2} H_{I} S^{2}+\cdots\right. \\
& \left.+\frac{1}{n !} H_{I} S^{n}\right)\left|\psi_{0}\right\rangle=0,
\end{aligned}
$$

where $E_{n}$ is defined by Eq. (4.17).

The difference between the variational and H-nijou equations is given by $\Delta_{n}$

$$
\begin{aligned}
\Delta_{n}^{I}\left(\left\{C_{I}\right\}\right)= & \left\langle\psi_{n}\right|\left(H-E_{n}\right)\left\{\frac{1}{2}\left(H_{I} S-S H_{I}\right)+\frac{1}{3 !}\left(2 H_{I} S^{2}\right.\right. \\
& \left.-S H_{I} S-S^{2} H_{I}\right)+\cdots+\frac{1}{n !}\left[(n-1) H_{I} S^{n-1}\right. \\
& \left.\left.-\sum_{m=2}^{n} S^{m-1} H_{I} S^{n-m}\right]\right\}\left|\psi_{0}\right\rangle \\
= & \left\langle\psi_{n}\right|\left(H-E_{n}\right)\left\{\frac{1}{2}\left[H_{I}, S\right]+\frac{1}{3 !}\left(2\left[H_{I}, S\right] S\right.\right. \\
& \left.+S\left[H_{I}, S\right]\right)+\cdots+\frac{1}{n !} \sum_{m=1}^{n-1}(n-m) S^{m-1} \\
& \left.\times\left[H_{I}, S\right] S^{n-m-1}\right\}\left|\psi_{0}\right\rangle .
\end{aligned}
$$

Since $\psi_{n}$ is approximate, $\Delta_{n}^{I}$ is not necessarily zero, but it should be small for good $\psi_{n}$ : It gives a measure how good is the quality of the calculated $\psi_{n}$. The smallness of $\Delta_{n}^{I}$ would be due to case B of the previous section: The $S$ and $H_{I}$ would not commute and all $C_{I}$ would not be equal. When $\Delta_{n}^{I}=0$, the difference between $F_{n}^{I}$ and $G_{n}^{I}$ becomes

$$
F_{n}^{I}-G_{n}^{I}=\left\langle\psi_{n}\left|\frac{1}{n !} H_{I} S^{n}\right| \psi_{0}\right\rangle
$$

The energy calculated by the variational method is always lower than that calculated by the H-nijou method. 


\section{PERFORMANCE OF SECC AND ECC2 FOR HARMONIC OSCILLATOR}

We examine here the performance of the SECC by applying it to a simple example, one-dimensional harmonic oscillator.

The harmonic oscillator with the force constant $k$ and the reduced mass $\mu$ is defined by the Schrödinger equation

$$
\left(-\frac{h^{2}}{8 \pi^{2} \mu} \frac{d^{2}}{d y^{2}}+\frac{1}{2} k y^{2}\right) \psi=E \psi .
$$

Replacing the constants by

$$
\beta^{4}=\frac{h^{2}}{4 \pi^{2} k \mu}, \quad \varepsilon=\frac{8 \pi^{2} \beta^{2} \mu E}{h^{2}},
$$

and the variable by

$$
y=\beta x,
$$

the Hamiltonian is written as

$$
H=-\frac{d^{2}}{d x^{2}}+x^{2},
$$

and the Schrödinger equation (5.1) is rewritten as

$$
\left(-\frac{d^{2}}{d x^{2}}+x^{2}\right) \psi=\varepsilon \psi
$$

The two lowest solutions of this equation are

$$
\psi_{g}=\pi^{-1 / 4} \exp \left(-\frac{1}{2} x^{2}\right), \quad \varepsilon_{g}=1,
$$

and

$$
\psi_{e}=\sqrt{2} \pi^{-1 / 4} x \exp \left(-\frac{1}{2} x^{2}\right), \quad \varepsilon_{e}=3 .
$$

We take

$$
\psi_{0}=\exp \left(-\alpha x^{2}\right),
$$

with $\alpha=0.55$ and 0.60 as our reference functions. $\alpha=0.5$ is exact solution. The energy expectation value of the reference function $\psi_{0}$ is

$$
\varepsilon_{0}=\left(1+4 \alpha^{2}\right) / 4 \alpha,
$$

TABLE I. Ground state of the harmonic oscillator by the variational method for the truncated SECC.

\begin{tabular}{rccccc}
\hline \hline & \multicolumn{2}{c}{$\alpha=0.55$} & & \multicolumn{2}{c}{$\alpha=0.60$} \\
\cline { 2 - 3 } \cline { 5 - 6 }$n$ & $C_{\text {opt }}$ & $E_{\text {opt }}$ & & $C_{\text {opt }}$ & $E_{\text {opt }}$ \\
\hline 0 & $\ldots$ & 1.004545 & & $\ldots$ & 1.016667 \\
1 & -0.1989 & 1.000015 & & -0.1961 & 1.000203 \\
2 & -0.1790 & 1.001664 & & -0.1760 & 1.006160 \\
3 & -0.3091 & 1.000077 & & -0.2887 & 1.000840 \\
4 & -0.2941 & 1.000639 & & -0.2781 & 1.002515 \\
5 & -0.3808 & 1.000175 & & -0.3344 & 1.001197 \\
6 & -0.3810 & 1.000288 & & -0.3427 & 1.001334 \\
7 & -0.4232 & 1.000173 & & -0.3681 & 1.001007 \\
8 & -0.4378 & 1.000167 & & -0.3817 & 1.000920 \\
9 & -0.4607 & 1.000133 & & -0.3965 & 1.000801 \\
10 & -0.4763 & 1.000118 & & -0.4082 & 1.000726 \\
15 & -0.5383 & 1.000069 & & -0.4513 & 1.000497 \\
20 & -0.5771 & 1.000050 & & -0.4779 & 1.000394 \\
30 & -0.6237 & 1.000033 & & -0.5093 & 1.000300 \\
\hline \hline
\end{tabular}

which has the minimum value 1.0 at $\alpha=0.5$, a consequence of the variational principle.

\section{A. SECC}

We now apply SECC first to the ground state. We use the SECC given by Eq. (3.14). A single variable $C$ is included in the $S$ operator as

$$
S=C\left(-\frac{d^{2}}{d x^{2}}+x^{2}\right)
$$

We have applied the variational method and the $f(C)$-minimization method to the truncated $\psi_{n}$ for $n$

\begin{tabular}{|c|c|c|c|c|c|c|}
\hline \multirow[b]{2}{*}{$n$} & \multicolumn{3}{|c|}{$\alpha=0.55$} & \multicolumn{3}{|c|}{$\alpha=0.60$} \\
\hline & $C_{\mathrm{opt}}$ & $E_{\mathrm{opt}}$ & $f(C)$ & $C_{\mathrm{opt}}$ & $E_{\mathrm{opt}}$ & $f(C)$ \\
\hline 0 & $\ldots$ & 1.004545 & $\ldots$ & $\ldots$ & 1.016667 & $\ldots$ \\
\hline 1 & -0.1981 & 1.000016 & $1.3 \times 10^{-4}$ & -0.1931 & 1.000209 & $1.7 \times 10^{-3}$ \\
\hline 2 & -0.1969 & 1.001691 & $7.8 \times 10^{-3}$ & -0.1896 & 1.006221 & $2.8 \times 10^{-2}$ \\
\hline 3 & -0.3021 & 1.000081 & $5.1 \times 10^{-4}$ & -0.2737 & 1.000921 & $5.0 \times 10^{-3}$ \\
\hline 4 & -0.3007 & 1.000641 & $2.5 \times 10^{-3}$ & -0.2749 & 1.002517 & $1.0 \times 10^{-2}$ \\
\hline 5 & -0.3638 & 1.000188 & $7.9 \times 10^{-4}$ & -0.3155 & 1.001270 & $5.2 \times 10^{-3}$ \\
\hline 6 & -0.3736 & 1.000290 & $1.0 \times 10^{-3}$ & -0.3281 & 1.001370 & $5.2 \times 10^{-3}$ \\
\hline 7 & -0.4062 & 1.000180 & $6.3 \times 10^{-4}$ & -0.3490 & 1.001062 & $3.9 \times 10^{-3}$ \\
\hline 8 & -0.4228 & 1.000172 & $5.7 \times 10^{-4}$ & -0.3627 & 1.000968 & $3.5 \times 10^{-3}$ \\
\hline 9 & -0.4427 & 1.000139 & $4.5 \times 10^{-4}$ & -0.3764 & 1.000851 & $2.9 \times 10^{-3}$ \\
\hline 10 & -0.4580 & 1.000124 & $3.8 \times 10^{-4}$ & -0.3878 & 1.000774 & $2.6 \times 10^{-3}$ \\
\hline 15 & -0.5178 & 1.000074 & $2.0 \times 10^{-4}$ & -0.4305 & 1.000539 & $1.6 \times 10^{-3}$ \\
\hline 20 & -0.5566 & 1.000054 & $1.3 \times 10^{-4}$ & -0.4579 & 1.000429 & $1.2 \times 10^{-3}$ \\
\hline 30 & -0.6046 & 1.000036 & $8.0 \times 10^{-5}$ & -0.4915 & 1.000326 & $8.4 \times 10^{-4}$ \\
\hline
\end{tabular}
$=1-30$. The calculations have been performed using MAPLE $6^{12}$ and the results are given in Tables I and II. The absolute value of $C, C_{\mathrm{opt}}$ of $\psi_{n}$ increases, as expected, as the number of terms, $n$ increases, and the corresponding energy $E_{\text {opt }}$ approaches the exact value 1.0. The magnitude of $C_{\mathrm{opt}}$ is, however, far from the negative infinity. Initially for small $n$, the results oscillate to some extent, but become monotonic for $n>6$. The convergence rate is faster for $\alpha=0.55$ than for

TABLE II. Ground state of the harmonic oscillator by the $f(C)$-minimization method for the truncated SECC. 
TABLE III. First excited state of the harmonic oscillator by the variational method for the truncated SECC.

\begin{tabular}{rccccc}
\hline \hline & \multicolumn{2}{c}{$\alpha=0.55$} & & \multicolumn{2}{c}{$\alpha=0.60$} \\
\cline { 2 - 3 } \cline { 5 - 6 }$n$ & $C_{\mathrm{opt}}$ & $E_{\mathrm{opt}}$ & & $C_{\mathrm{opt}}$ & $E_{\mathrm{opt}}$ \\
\hline 0 & $\ldots$ & 3.013636 & & $\ldots$ & 3.05 \\
1 & -0.1419 & 3.000077 & & -0.1396 & 3.001000 \\
2 & -0.1130 & 3.007207 & & -0.1115 & 3.026552 \\
3 & -0.2230 & 3.000288 & & -0.2121 & 3.003280 \\
4 & -0.1983 & 3.003646 & & -0.1925 & 3.013703 \\
5 & -0.2885 & 3.000703 & & -0.2584 & 3.005412 \\
6 & -0.2754 & 3.001915 & & -0.2573 & 3.007716 \\
7 & -0.3321 & 3.000904 & & -0.2914 & 3.005168 \\
8 & -0.3365 & 3.001109 & & -0.3020 & 3.005098 \\
9 & -0.3676 & 3.000783 & & -0.3209 & 3.004223 \\
10 & -0.3808 & 3.000742 & & -0.3333 & 3.003860 \\
15 & -0.4528 & 3.000402 & & -0.3854 & 3.002481 \\
20 & -0.4998 & 3.000272 & & -0.4193 & 3.001865 \\
30 & -0.5594 & 3.000166 & & -0.4615 & 3.001307 \\
\hline \hline
\end{tabular}

$\alpha=0.60$, because the quality of the reference function is better for $\alpha=0.55$. The variational results given in Table I and the $f(C)$-minimization results given in Table II show similar behaviors. The energy of the former is always lower than that of the latter, but the difference becomes smaller as $n$ increases. The value of $f(C)$, which must be positive, gradually approaches zero from above. The convergence rate is slow in both methods: we do not yet reach the convergence especially for $\alpha=0.60$. But, we can conclude that the SECC converges to the exact value for both the results given in Tables I and II.

The SECC has also been applied to the first excited state of the harmonic oscillator for which the exact solution is given by Eq. (5.7). Similarly to the ground state, we use the SECC given by Eq. (3.14) and we take the reference function

$$
\psi_{0}=x \exp \left(-\alpha x^{2}\right),
$$

with $\alpha=0.55$ and 0.60 . This reference function is ungerade for the inversion symmetry as the exact wave function: the first excited state is the ground state of the ungerade symmetry. The energy of the reference function is

$$
\varepsilon_{1}=3\left(1+4 \alpha^{2}\right) / 4 \alpha,
$$

which has a minimum again at $\alpha=0.5$. We have applied the variational method to the truncated $\psi_{n}$ of the SECC using
MAPLE 6 and the results are shown in Table III. The convergence behavior of the excited state is similar to that of the ground state. It initially oscillates but becomes monotonic for $n>8$. The value of $C_{\text {opt }}$ increases as $n$ increases and $E_{\text {opt }}$ approaches the exact value 3.0, though the convergence rate is slow.

In Tables I to III, the result for $\psi_{1}$ is remarkably good. It is essentially the result of the first iteration of the SICI method. ${ }^{2}$ Since every step is variational in the ICI method and since the variable is reoptimized in each iteration step, the convergence of the SICI is expected to be faster than that of the SECC, if the SECC is solved in the expansion method as given here.

\section{B. ECC2}

We next apply ECC2 to the ground state of the harmonic oscillator. Two variables $C_{K}$ and $C_{P}$ are associated to the kinetic and potential operators as

$$
S=-C_{K} \frac{d^{2}}{d x^{2}}+C_{P} x^{2} .
$$

Note that $S$ and $H$ do not commute as far as $C_{K}$ is not equal to $C_{P}$. The variational method and the $\mathrm{H}$-nijou method are different in ECC2.

We first apply the variational method. We calculate the $\mathrm{ECC} 2$ wave function truncated at $n=2$ and 3 and summarize the results in Table IV. $C_{K}$ and $C_{P}$ in Table IV are accurate only to five decimal figures. The result for $n=1$ is rather special. At $n=1$, the ECC2 is identical with the first iteration of ICI2, and in this example of harmonic oscillator, the first iteration of ICI2 is also just identical with the first iteration of SICI or ICI1 because $\psi_{0},-\left(d^{2} / d x^{2}\right) \psi_{0}$, and $x^{2} \psi_{0}$ are not linearly independent. Thus, the ECC2 result at $n=1$ is identical with the SECC result at $n=1$ given in Table I.

From Table IV, we are surprised at the good convergence of the ECC2 wave function. Even at $n=2$, the energy converges very nicely to 1.0 , and at $n=3$, the results are already very accurate. This is in sharp contrast to the result of SECC given in Table I, where the convergence is very slow and the energy is still considerably different from 1.0 even at $n=30$ for $\alpha=0.60$. The optimal $C_{K}$ and $C_{P}$ of ECC2 are not equal but differ even in sign. Therefore, this ECC2 solution is different from the SECC solution. Further, the

\begin{tabular}{|c|c|c|c|c|c|}
\hline & & \multicolumn{2}{|c|}{$n=2$} & \multicolumn{2}{|c|}{$n=3$} \\
\hline & & $\alpha=0.55$ & $\alpha=0.60$ & $\alpha=0.55$ & $\alpha=0.60$ \\
\hline \multirow[t]{2}{*}{ Optimal } & $C_{K}$ & -0.02010 & -0.03641 & -0.02010 & -0.03662 \\
\hline & $C_{P}$ & +0.02792 & +0.05650 & +0.02785 & +0.05580 \\
\hline \multirow{3}{*}{$\begin{array}{l}E_{n} \\
G_{n}^{I}\end{array}$} & & 1.0000000440 & 1.0000021064 & 1.00000000021 & 1.00000003127 \\
\hline & $I=K$ & $0.2270 \times 10^{-5}$ & $-1.1605 \times 10^{-5}$ & $1.2298 \times 10^{-5}$ & $1.2517 \times 10^{-5}$ \\
\hline & $I=P$ & $0.2647 \times 10^{-5}$ & $0.3959 \times 10^{-5}$ & $-1.1166 \times 10^{-5}$ & $1.0381 \times 10^{-5}$ \\
\hline \multirow[t]{2}{*}{$\Delta_{n}^{I}$} & $I=K$ & $0.0427 \times 10^{-5}$ & $-0.2290 \times 10^{-5}$ & $-0.0648 \times 10^{-5}$ & $0.1253 \times 10^{-5}$ \\
\hline & $I=P$ & $0.0307 \times 10^{-5}$ & $-0.1475 \times 10^{-5}$ & $-0.0594 \times 10^{-5}$ & $0.2094 \times 10^{-5}$ \\
\hline \multirow[t]{2}{*}{$\partial E_{n} / \partial C_{I}$} & $I=K$ & $-0.2672 \times 10^{-5}$ & $1.4179 \times 10^{-5}$ & $1.4474 \times 10^{-5}$ & $1.5306 \times 10^{-5}$ \\
\hline & $I=P$ & $0.3115 \times 10^{-5}$ & $0.4837 \times 10^{-5}$ & $-1.3142 \times 10^{-5}$ & $1.2694 \times 10^{-5}$ \\
\hline
\end{tabular}

TABLE IV. Truncated ECC2 for harmonic oscillator calculated by the variational method. At $n=1, C_{K}=C_{P}$ $=-0.1989$ and $E_{1}=1.000015$ for $\alpha=0.55$, and $C_{K}=C_{P}=-0.1961$ and $E_{1}=1.000203$ for $\alpha=0.60$. 
TABLE V. Truncated ECC2 for harmonic oscillator calculated by the H-nijou method. At $n=1, C_{K}=C_{P}$ $=-0.1989$ and $E_{1}=1.000015$ for $\alpha=0.55$, and $C_{K}=C_{P}=-0.1961$ and $E_{1}=1.000203$ for $\alpha=0.60$.

\begin{tabular}{|c|c|c|c|c|c|}
\hline & & \multicolumn{2}{|c|}{$n=2$} & \multicolumn{2}{|c|}{$n=3$} \\
\hline & & $\alpha=0.55$ & $\alpha=0.60$ & $\alpha=0.55$ & $\alpha=0.60$ \\
\hline \multirow[t]{2}{*}{ Optimal } & $C_{K}$ & -0.02010 & -0.03641 & -0.02010 & -0.03662 \\
\hline & $C_{P}$ & +0.02792 & +0.05653 & +0.02785 & +0.05580 \\
\hline$E_{n}$ & & 1.0000000440 & 1.0000021081 & 1.00000000021 & 1.00000003127 \\
\hline \multirow{2}{*}{$F_{n}^{I}$} & $I=K$ & $0.2624 \times 10^{-5}$ & $3.9591 \times 10^{-5}$ & $1.1669 \times 10^{-5}$ & $-0.8661 \times 10^{-5}$ \\
\hline & $I=P$ & $-0.1738 \times 10^{-5}$ & $0.2046 \times 10^{-5}$ & $-1.1665 \times 10^{-5}$ & $0.9328 \times 10^{-5}$ \\
\hline$n$ th-order & $I=K$ & $0.4468 \times 10^{-5}$ & $1.4203 \times 10^{-4}$ & $0.2020 \times 10^{-7}$ & $0.2602 \times 10^{-5}$ \\
\hline term $^{\mathrm{a}}$ & $I=P$ & $-0.4692 \times 10^{-5}$ & $-0.8852 \times 10^{-4}$ & $-0.3100 \times 10^{-7}$ & $-0.1876 \times 10^{-5}$ \\
\hline
\end{tabular}

${ }^{\mathrm{a}}$ This is the term defined by Eq. (4.20).

values of $\Delta_{n}^{K}$ and $\Delta_{n}^{P}$ are remarkably very small, showing that the variational solution is very close to the H-nijou solution. This is confirmed from Table $\mathrm{V}$, in which the results of the H-nijou method are summarized. This smallness of $\Delta_{n}^{l}$ is attained though $S$ and $H$ do not commute. This is case B, explained in the previous section. The optimal character of the ECC2 results shown in Table IV is confirmed from the smallness of the values of $G_{n}^{I}(I=K, P)$ and $\partial E_{n} / \partial C_{I}$ at the optimal values of $C_{K}$ and $C_{P}$.

Table $\mathrm{V}$ shows the result of the $\mathrm{H}$-nijou method applied to $n=2$ and 3 of ECC2. The optimal values of $C_{K}$ and $C_{P}$ are very close to those of the variational method. Actually, the H-nijou results are the same as the variational results except for the case of $\alpha=0.60$ and $n=2$, where the secondorder term in the H-nijou integral $F_{n}^{I}$, which is the difference between $F_{n}^{I}$ and $G_{n}^{I}$ as seen from Eq. (4.20), is the little bit large so that the variational result and the $\mathrm{H}$-nijou result become different. Note that this difference is not due to a large $\Delta_{n}^{I}$ value. It is also small even in this case. The optimal character of $C_{K}$ and $C_{P}$ is seen from the smallness of the value $F_{n}^{I}$.

The results of ECC2 given in this section very much encourage the ECCND approach for more realistic systems. The key is a smallness of the $\Delta_{n}^{I}$ values that guarantees the high quality of the calculated results. This high quality of the calculated results is certainly due to the exactness of the structure of the ECCND wave function.

\section{CONCLUSION}

In this series of papers, ${ }^{1,2}$ we have investigated the structure of the exact wave function and obtained several expressions of the exact wave function that includes much smaller number of variables than $M_{\text {full-CI }}$ given by Eq. (1.3). The number of variables is from unity to $M_{\mathrm{GSD}}$, for example, depending on the method of division of the Hamiltonian. Since the exact wave function is unique, these different expressions represent the different aspects of a single reality, the exact wave function. These expressions give the bases for the further studies of the exact wave function from both theoretical and numerical aspects. They will also give a hint in the study of the different frameworks of the quantum mechanics, like DFT and DMT (density matrix theory). ${ }^{13}$

In this paper, we have examined the exponential ansatz in the light of the general theorem given in Paper II. As motivated in Paper I, we could prove that the simplest extended coupled cluster (SECC) has the structure of the exact wave function: it becomes exact when solved variationally or by the H-nijou method. This fact sheds light on the possibility of the ECCND wave function in general. Since even the simplest ECC is exact, the more general ECCND should also be exact. Then, both the variational equation and the H-nijou equation give the exact wave function when applied to ECCND. For the exact solution, the difference between the variational equation and the H-nijou equation should vanish and is satisfied as an integral relation given by Eq. (3.29). Thus, the noncommuting problem pointed out in Paper I disappears. ${ }^{9}$ Further, ECCND $\left(N_{D} \geqslant 2\right)$ may give a more rapidly converging solution than SECC. Because of its nonlinear character, ECCND may give the exact solution at the sets of variables different from SECC. We thus conclude that the ECCND is exact for all $N_{D}$. ECCND gives an explicit expression of the wave operator $W$ defined by Eq. (1.5). As a special case, CCGSD is also exact: a statement in Paper I must be corrected. ${ }^{9}$ The ECCND and CCGSD must be solved by the variational method or by the H-nijou method. Nooijen ${ }^{4}$ considered solving the CCGSD using the density equation, ${ }^{5}$ which belongs to the H-nijou method: this is a clever idea to give the exact solution.

We have summarized the method of solving the SECC and ECCND for the $n$ th-order truncated form. The variational equation and the $\mathrm{H}$-nijou equation are different in this case, but the difference should be small: it gives a measure of the quality of the calculated result.

The SECC and the ECCND are applied in their truncated form to the simple example of harmonic oscillator. The SECC certainly gave the exact wave function starting from approximate reference functions for both the ground- and first-excited states, though the convergence rates were rather slow. On the other hand, ECC2, having different variables for kinetic and potential operators, gave quite a rapid convergence. The variational method and the H-nijou method gave essentially the same results: $\Delta_{n}^{I}=0$ were essentially satisfied. This good performance of the ECCND method certainly reflects its exact structure as a wave function. CCGSD is also important as a special case of ECCND. Head-Gordon and his co-workers recently studied variational coupled cluster in some detail and reported several nice results. ${ }^{14}$ Based on the present result, we believe that the study of ECCND 
would certainly open up a new wave in the study of the exact wave function.

\section{ACKNOWLEDGMENTS}

The author thanks Professor E. R. Davidson for some e-mail discussions and Dr. M. Hada and Dr. M. Ehara for several useful conversations and help. The discussions with Professor D. Mukherjee were also helpful in realizing the normal-ordered exponential ansatz. This study has been supported financially by the special fund from the Ministry of Education, Science, Culture, and Sports of Japan.

${ }^{1}$ H. Nakatsuji, J. Chem. Phys. 113, 2949 (2000).

${ }^{2}$ H. Nakatsuji and E. R. Davidson, J. Chem. Phys. 5, 2000 (2001).

${ }^{3}$ P. A. M. Dirac, Proc. R. Soc. London, Ser. A 123, 714 (1929).

${ }^{4}$ M. Nooijen, Phys. Rev. Lett. 84, 2108 (2000).

${ }^{5}$ H. Nakatsuji, Phys. Rev. A 14, 41 (1976).
${ }^{6}$ R. G. Parr and W. Yang, Density-Functional Theory of Atoms and Molecules (Springer, New York, 1989).

${ }^{7}$ T. J. Park and J. C. Light, J. Chem. Phys. 85, 5870 (1986).

${ }^{8}$ R. Kosloff and H. Tal-Ezer, Chem. Phys. Lett. 127, 223 (1986); R. Kosloff, J. Phys. Chem. 92, 2087 (1988).

${ }^{9}$ In the previous paper (Ref. 1), we wrote, "Therefore, the variational CCGSD is not exact." This statement should be read, "Therefore, the trial form of the CCGSD is not guaranteed to be exact." But, the fact that the SECC has a structure of the exact wave function implies that the CCGSD is also exact when it is solved by the variational or H-nijou method.

${ }^{10}$ I. Lindgren, Int. J. Quantum Chem. S12, 33 (1978).

${ }^{11}$ I. Lindgren and D. Mukherjee, Phys. Rep. 151, 93 (1987); U. S. Mahapatra, B. Datta, B. Bandyopadhyay, and D. Mukherjee, Adv. Quantum Chem. 30, 163 (1998)

${ }^{12}$ M. B. Monagan, K. O. Geddes, K. M. Heal, G. Labahn, S. M. Vorkoetter, and J. McCarron, MAPLE 6, Waterloo Maple, Inc., Waterloo, Canada.

${ }^{13}$ Many-Electron Densities and Reduced Density Matrices, edited by J. Cioslowski (Kluwer Academic, New York, 2000).

${ }^{14}$ T. V. Voorhis and M. Head-Gordon, J. Chem. Phys. 113, 8873 (2000). 